\title{
Sex differences in innate and adaptive neural oscillatory patterns link resilience and susceptibility to chronic stress in rats
}

\author{
Rachel-Karson Thériault, MSc; Joshua D. Manduca, BSc; Melissa L. Perreault, PhD
}

\begin{abstract}
Background: Major depressive disorder is a chronic illness with a higher incidence in women. Dysregulated neural oscillatory activity is an emerging mechanism thought to underlie major depressive disorder, but whether sex differences in these rhythms contribute to the development of symptoms is unknown. Methods: We exposed male and female rats to chronic unpredictable stress and characterized them as stress-resilient or stress-susceptible based on behavioural output in the forced swim test and the sucrose preference test. To identify sex-specific neural oscillatory patterns associated with stress response, we recorded local field potentials from the prefrontal cortex, cingulate cortex, nucleus accumbens and dorsal hippocampus throughout stress exposure. Results: At baseline, female stressresilient rats innately exhibited higher theta coherence in hippocampal connections compared with stress-susceptible female rats. Following stress exposure, additional oscillatory changes manifested: stress-resilient females were characterized by increased dorsal hippocampal theta power and cortical gamma power, and stress-resilient males were characterized by a widespread increase in high gamma coherence. In stress-susceptible animals, we observed a pattern of increased delta and reduced theta power; the changes were restricted to the cingulate cortex and dorsal hippocampus in males but occurred globally in females. Finally, stress exposure was accompanied by the time-dependent recruitment of specific neural pathways, which culminated in system-wide changes that temporally coincided with the onset of depression-like behaviour. Limitations: We could not establish causality between the electrophysiological changes and behaviours with the methodology we employed. Conclusion: Sex-specific neurophysiological patterns can function as early markers for stress vulnerability and the onset of depression-like behaviours in rats.
\end{abstract}

\section{Introduction}

About $10 \%$ of the world population has major depressive disorder (MDD), and prevalence is 2 times greater in women than in men. ${ }^{1,2}$ Chronic stress is an established risk factor for the development of MDD: ${ }^{3}$ clinical and preclinical studies have shown that females have greater behavioural sensitivity to chronic stress exposure. ${ }^{4-7}$ This suggests that sex differences exist in the stress-response neural circuitry linked to the development of depression symptoms. ${ }^{8}$ However, despite the known predominance of depression in women, most studies that have examined the influence of stress on depression have not employed sex as a factor. Thus, the mechanisms that contribute to the increased vulnerability of females to depression remain poorly understood.

The dysregulation of circuit function in the putative depression network has been linked to depression symptoms and antidepressant responsiveness; many studies have focused on low-frequency changes. ${ }^{9}$ Electroencephalography
(EEG) studies most commonly report frontal and parietal alpha asymmetries as potential endophenotypes of MDD, ${ }^{10-13}$ because these patterns distinguish currently symptomatic and remitted patients from those with no history of depression. ${ }^{11,12,14,15}$ Alterations in midline theta activity in MDD are also commonly reported, although results are inconsistent: studies have shown increased activity, ${ }^{16,17}$ decreased activity $^{18-21}$ or no change. ${ }^{22,23}$ A relationship between midline theta activity and antidepressant treatment response also exists, but with the same discrepant findings. ${ }^{17,20,24-26}$ Gamma-band deficits have also been implicated as a potential marker of $\mathrm{MDD}^{27-29}$ and therapeutic antidepressant efficacy, ${ }^{30-32}$ and increased gamma activity has been associated with the remission of depression symptoms. ${ }^{30-32}$

Few studies have considered the role of sex in dysregulated network activity in MDD, and of those that exist, there appears to be no consensus. For example, EEG studies have reported that the frontal alpha asymmetry is present solely in women with MDD, ${ }^{33-36}$ but others have shown

Correspondence to: M. L. Perreault, Department of Molecular and Cellular Biology, 50 Stone Rd. E., University of Guelph, Guelph, ON N1G 2W1; perreaum @uoguelph.ca

Submitted Jul. 3, 2020; Revised Sep. 21, 2020; Accepted Oct. 22, 2020

DOI: 10.1503/jpn.200117 
it in both sexes. ${ }^{16,37}$ It has also been reported that only women have a positive association between frontal asymmetry and the severity of MDD. ${ }^{15,33}$ Similarly, whereas 1 study found that only women with MDD had increased right (versus left) parietal activity, ${ }^{16}$ another found no differences in parietal activation in women with MDD compared to healthy controls. ${ }^{38}$ Therefore, although evidence suggests that low-frequency oscillatory activity is a potential important contributor to depression, the exact nature of the relationship remains uncertain.

We hypothesized that innate and stress-induced changes in low-frequency neural oscillatory activity exist that are unique to each sex and that contribute to depression vulnerability. To investigate this hypothesis, we evaluated sex differences in circuit function adaptations induced by exposure to chronic unpredictable stress (CUS) in rats and discerned the ability of these functional signatures to serve as early markers of stress resilience or susceptibility.

\section{Methods}

\section{Animals}

We used adult male and female Wistar rats (Charles River) that weighed 175-225 g ( 6 weeks of age) at the beginning of the experiment, and we completed the experiment using 2 independent cohorts of rats. Animals were weighed weekly and housed singly in the same temperature-controlled colony room (temperature $21^{\circ} \mathrm{C}$, humidity $30 \%-45 \%$ ), maintained on a 12-hour reverse light-dark cycle unless otherwise noted. We conducted behavioural testing during the dark phase of the day-night cycle. During the experiment, 15\% of animals had to be removed because of head-cap detachment before study completion. All procedures were approved by the Animal Care Committee of the University of Guelph and carried out in accordance with the recommendations of the Canadian Council on Animal Care (AUP\# 3788).

\section{Surgeries}

Rats were anesthetized with isoflurane, given the analgesic carprofen (5 mg/ $\mathrm{kg}$, s.c.) and secured in a stereotaxic frame. Custom electrode microarrays, built using prefabricated Delrin templates and polyimide-insulated stainless steel wires (A-M Systems: 791600, 0.008"), were implanted bilaterally into the prefrontal cortex (PFC; anterior-posterior [AP] $+3.24 \mathrm{~mm}$, medial-lateral [ML] $\pm 0.6 \mathrm{~mm}$, dorsal-ventral [DV] $-3.8 \mathrm{~mm})$, the cingulate cortex $(\mathrm{Cg} ; \mathrm{AP}+1.9 \mathrm{~mm}, \mathrm{ML}$ $\pm 0.5 \mathrm{~mm}, \mathrm{DV}-2.8 \mathrm{~mm}$ ), the nucleus accumbens (NAc; AP $+1.9 \mathrm{~mm}, \mathrm{ML} \pm 1.2 \mathrm{~mm}, \mathrm{DV}-6.6 \mathrm{~mm})$ and the CA1 region of the dorsal hippocampus (dHIP; AP $-3.5 \mathrm{~mm}, \mathrm{ML} \pm 2.5 \mathrm{~mm}$, DV $-2.6 \mathrm{~mm}$ ). A ground screw was implanted into the skull behind $\lambda$, and additional anchor screws were attached to the skull and secured with dental cement. To verify electrode placements, lesions were produced using the Pulse Pal (v2.0; Sanworks) to deliver a $30 \mathrm{mV}$ single-train pulse through each electrode for visualization postmortem (Appendix 1, Fig. S1, available at jpn.ca/200117-a1).

\section{Electrophysiology}

We performed local field potential (LFP) recordings (Wireless 2100-System; Multichannel Systems) in awake, resting animals in clear plexiglass boxes $(45.7 \mathrm{~cm} \times 45.7 \mathrm{~cm} \times 45.7 \mathrm{~cm})$. Baseline recordings were collected 24 hours before the CUS procedure, and 3 times per week throughout the procedure, at a rate of 1000 samples per second. Because the potentials were not stimulus-induced and thus were consistent throughout the LFP recordings, we collected recordings for 30 minutes. We used routines from the Chronux software package for MATLAB (MathWorks) to analyze the spectral power in each region and the coherence between regions. We used 5-minute epochs, and epochs were segmented, detrended, denoised and low-pass-filtered to remove frequencies greater than $100 \mathrm{~Hz}$. We calculated continuous multitaper spectral power for the normalized data (to total spectral power) and coherence (tapers = [5 9]) for delta $(1-4 \mathrm{~Hz})$, theta $(>4-12 \mathrm{~Hz})$, beta $(>12-30 \mathrm{~Hz})$, low gamma $(>30-60 \mathrm{~Hz})$ and high gamma (> 60-100 Hz) unless otherwise stated.

\section{Estrous staging}

Female animals underwent noninvasive vaginal lavage (100-200 $\mu \mathrm{L} 0.9 \%$ saline) to determine their estrous stage at least 2 hours before all behavioural testing and LFP recordings. We collected baseline behaviours before the surgeries and reassessed behaviours weekly throughout the CUS procedure.

\section{Behaviour}

We used the forced swim test to assess behavioural despair, performed as previously described ${ }^{39}$ For the pretest, animals were placed in a plexiglass cylinder, filled with $24 \pm 1^{\circ} \mathrm{C}$ water to the height of $35 \mathrm{~cm}$, for 15 minutes. Then, 24 hours after the pretest, animals were once again placed in the waterfilled cylinder for 5 minutes. The water was changed after every animal. For all subsequent tests, we did not perform the pretest, as previously described ${ }^{40-42}$ At 5-second intervals, we measured the following parameters: climbing (both front paws breaking the surface of the water while attempting to jump out of the cylinder), swimming (movement of limbs paddling across the water surface) or immobility (passive floating with movements only necessary to keep nose above water). Scoring was inherently blind, because allocation of the experimental groups was not predetermined.

We performed testing in the elevated plus maze as previously described. ${ }^{43}$ Animals were placed in the centre square of the maze (head facing an open arm) and allowed to explore for 10 minutes. We recorded behaviour using ANY-maze software (Stoelting Co.), and we measured the number of open- and closed-arm entries and the total time spent in the open and closed arms.

We used the sucrose preference test to assess anhedonia. Prior to the first test, we placed 2 bottles of $1 \%$ (wt/vol) sucrose solution in each cage for 24 hours, to habituate the animals to the sucrose solution. For the following 24 hours, 
we replaced 1 bottle of sucrose solution with water. Once habituation was complete, animals were deprived of food and water for 24 hours, after which we conducted the sucrose preference test. Animals were given 2 preweighed bottles: 1 containing 1\% sucrose solution and 1 containing water. Bottles were counterbalanced between cages and switched after every measurement. The bottles were reweighed every hour for 3 hours. Then, we calculated the percent sucrose preference (volume sucrose solution consumed/total volume consumed $\times 100$ ).

\section{Chronic unpredictable stress}

The CUS animal model for the study of depression is the model system with the greatest validity and translational potential; ${ }^{44}$ we conducted it as previously described. ${ }^{45}$ Rats were exposed to CUS until approximately half within each sex exhibited a depression-like phenotype. Animals that developed a depression-like phenotype were considered stresssusceptible; they were characterized by a minimum $60 \%$ increase from baseline in forced swim test immobility and a $20 \%$ decrease from baseline in sucrose preference. ${ }^{46}$ Animals that did not show more than a $10 \%$ increase in forced swim test immobility or a $10 \%$ decrease in sucrose preference compared to baseline were considered stress-resilient. ${ }^{46}$ The division of animals into groups based on the aforementioned criteria was validated by confirming a bimodal distribution of the behavioural data. Because of the within-subjects design of the study, we did not use control animals. The protocol consisted of various uncontrollable, nondebilitating and inescapable physical, psychological and circadian stressors: cold exposure $\left(4^{\circ} \mathrm{C}, 1\right.$ hour), lights on and off intermittently (8 hours), cage tilt $\left(30-45^{\circ}, 8\right.$ hours), damp bedding $(500 \mathrm{~mL}$ water, $12-14$ hours), cold swim $\left(10-13^{\circ} \mathrm{C}, 5\right.$ minutes), reverse light cycle (24 hours) and food and water deprivation (24 hours). Stressors were given on a random and unpredictable schedule. The same stressor did not reoccur for a minimum of 48 hours, and stress schedules differed across each week. All animals were exposed to the same stress schedule. Environmental enrichment was removed from all cages at the start of the CUS protocol, because it has been shown to mitigate susceptibility to stress in rodents. ${ }^{47-49}$ For a full schedule of the stressors and experimental testing, see Appendix 1, Table S1.

\section{Statistical analyses}

We performed power calculations using preliminary behavioural data, because behaviour has greater variability than LFP data, and power was set to 0.95 for calculations. Prior to analyses, we assessed normality using the Shapiro-Wilk test. For the behavioural data, we used a 2-way repeatedmeasures analysis of variance (ANOVA), with resiliency as the between-subjects factor and time as the within-subjects factor, followed by planned comparisons with a Student $t$ test. Because we used behavioural testing to characterize resiliency or susceptibility to chronic stress within each sex, we did not include sex as a variable in the analyses.
We analyzed the LFP data using 3-way repeated-measures ANOVA, with sex and resiliency as between-subjects factors and time as the within-subjects factor. In case of significant main effects or interactions, we identified individual mean differences by paired or independent Student $t$ tests. To assess the temporality of the stress-induced electrophysiological changes, we used 2-way repeated-measures ANOVA with resiliency as the between-subjects factor and time as the within-subjects factor, followed by planned comparisons using paired $t$ tests. All LFP data underwent Bonferroni correction for multiple comparisons $(\alpha=0.0125)$ at the level of each frequency within each brain region. Findings that were more than 2 standard deviations above or below the mean were considered outliers and were not included in the analysis. Artifacts in channels were manually identified and also excluded from the data set. We performed correlation analyses using linear regression. Computations were performed using SPSS 25 (IBM), and data are presented as the mean \pm standard error of the mean.

\section{Results}

The full experimental timeline is shown in Figure 1A. At baseline, we found no sex differences in forced swim test immobility time, and female rats showed lower sucrose preference (Figure 1B and C). In the elevated plus maze, males exhibited greater anxiety-like behaviour than females (Figure 1D). A maximum of 3 weeks of CUS exposure induced a depressionlike phenotype in approximately half of the female rats $(n=$ 10 resilient, $n=9$ susceptible), whereas males required a maximum of 5 weeks of CUS exposure $(n=9$ resilient, $n=8$ susceptible; Appendix 1, Figure S2A and B). However, CUS did not alter anxiety responses (Appendix 1, Figure S2C). In females, no baseline behaviours were correlated with estrous cycle stage, and cycling was disrupted by week 2 of CUS in all females (Appendix 1, Figure S3A-C).

\section{Baseline sex-and resiliency-dependent differences in oscillatory activity}

An important role for neural oscillations in depression has been shown ${ }^{9}$ and yet, knowledge about sex differences in depression-associated oscillations is limited..$^{50}$ Therefore, we first characterized sex differences in baseline and stress-induced oscillatory power recorded from regions implicated in depression: PFC, Cg, NAc and dHIP. We found no baseline sex differences in spectral power recorded from the $\mathrm{PFC}$ or $\mathrm{Cg}$ (Figure $1 \mathrm{E}$ and F). In the NAc, female rats showed lower NAc beta power (Figure 1G) than males, and in the dHIP they displayed greater theta and beta power, and lower delta and high gamma power (Figure $1 \mathrm{H}$ ). Females had higher baseline coherence in the delta, theta and beta frequency bands for all regional connections, except PFC-dHIP and NAc-dHIP (Figure 1I-N). Estrous stage did not influence baseline spectral power or coherence (Appendix 1, Figure S3D-M).

We next examined baseline differences in oscillatory activity between animals categorized as stress-resilient or stress-susceptible to determine whether such differences 
A

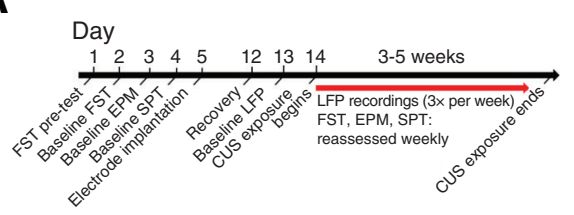

B

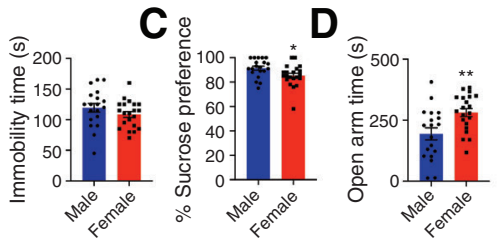

E PFC
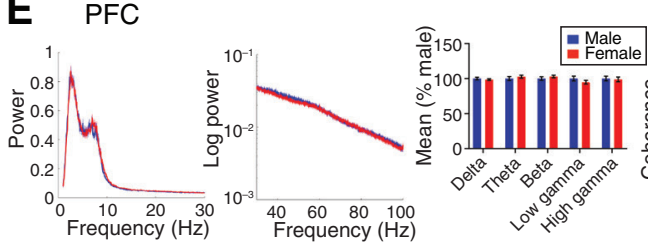

J PFC-NAC

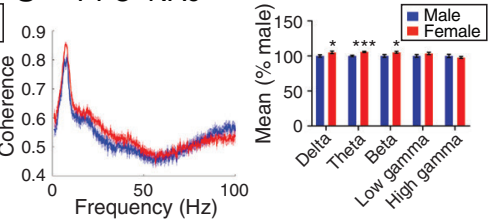

F $\mathrm{Cg}$
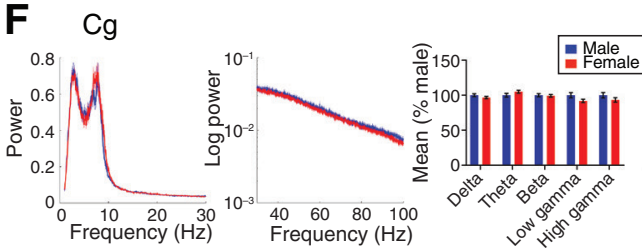

K PFC-dHIP
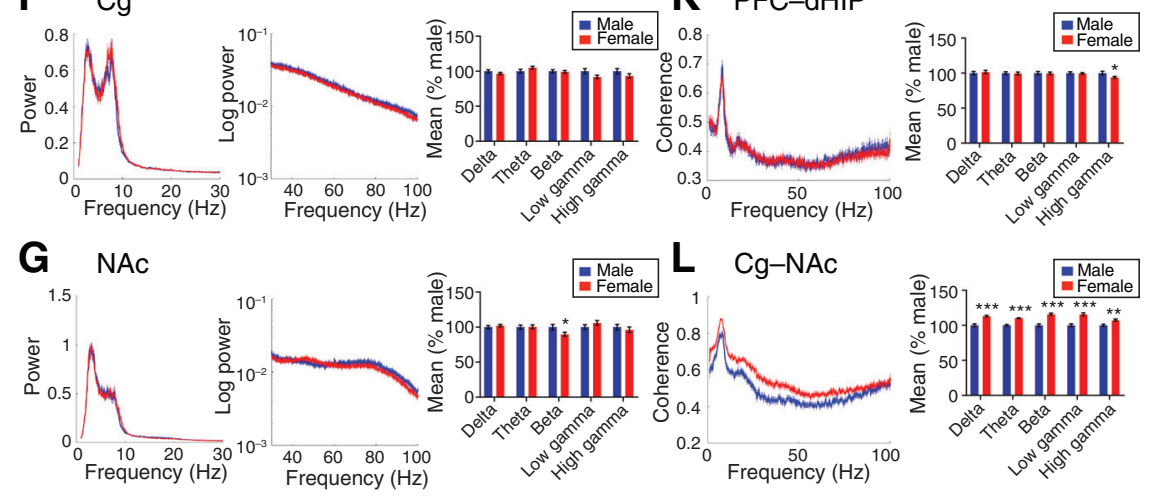

L Cg-NAC
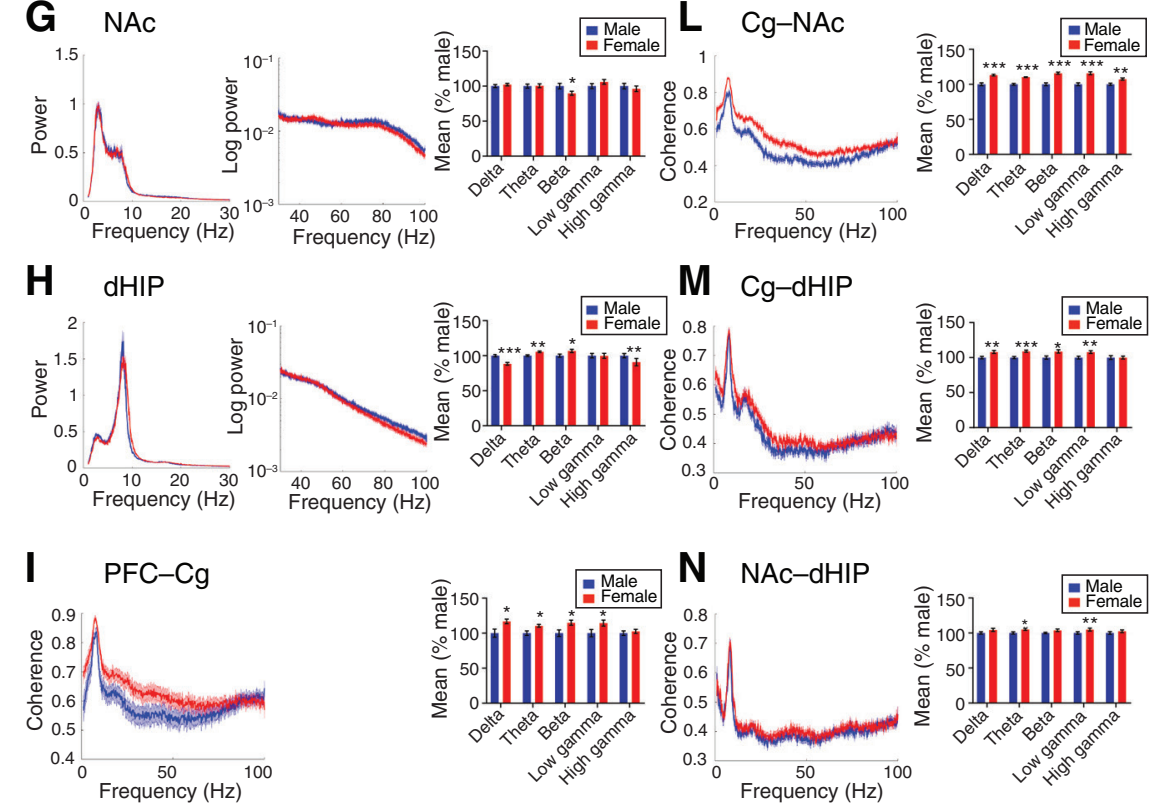

Fig. 1: Baseline sex differences in depression-like behaviours and oscillatory activity in rats. (A) The full experimental timeline is shown. (BD) No sex differences in time spent immobile in the FST at baseline were evident; however, females showed a lower percent sucrose preference in the SPT and spent more time in the open arms of the EPM. (E-H) Power spectra (left and centre panels) and quantification of power spectra (right panel) at each frequency are shown. We observed no sex differences in mean power spectra in the PFC or the Cg. In the $\mathrm{NAc}$, female rats had lower beta power, than males. In the dHIP, female rats innately had lower delta and high gamma power than male rats, as well as greater theta and beta power. (I-N) Coherence spectra (left panel) and quantification (right panel) at each frequency. Baseline coherence in the PFC-Cg of females was greater in the delta, theta, beta and low gamma frequency bands compared to males. In the PFC$\mathrm{NAc}$, coherence in the delta, theta and beta frequency bands was higher in female rats than in male rats. In the PFC-dHIP, males innately had greater high gamma coherence. Baseline coherence of all frequency bands in the $\mathrm{Cg}-\mathrm{NAc}$ were higher in females than males. In the Cg$\mathrm{dHIP}$, coherence was greater in females in all frequency bands except high gamma. Baseline theta frequency coherence was higher in females in the NAc-dHIP. Power and coherence spectra are presented as normalized data, with jackknife estimates of SEM shown as shaded areas. Quantified power spectra and coherence are presented as percent difference from males \pm SEM. A Student $t$ test was used for all analyses. ${ }^{\star} p<0.05,{ }^{\star \star} p<0.01,{ }^{* \star \star} p<0.001$, compared with males. $n=17$ males and $n=19$ females, 2 electrodes per region per rat. Cg $=$ cingulate cortex; CUS = chronic unpredictable stress; dHIP = dorsal hippocampus; EPM = elevated plus maze; FST = forced swim test; LFP = local field potential; NAc = nucleus accumbens; PFC = prefrontal cortex; $\mathrm{SEM}=$ standard error of the mean; $\mathrm{SPT}=$ sucrose preference test. 
A PFC-dHIP
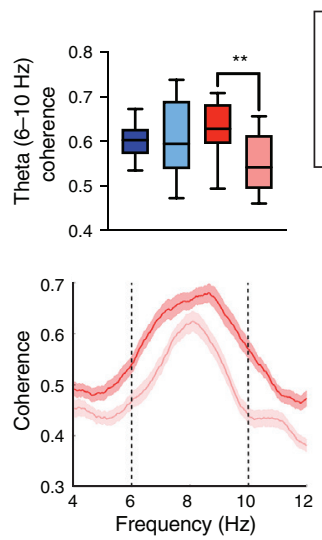

D PFC
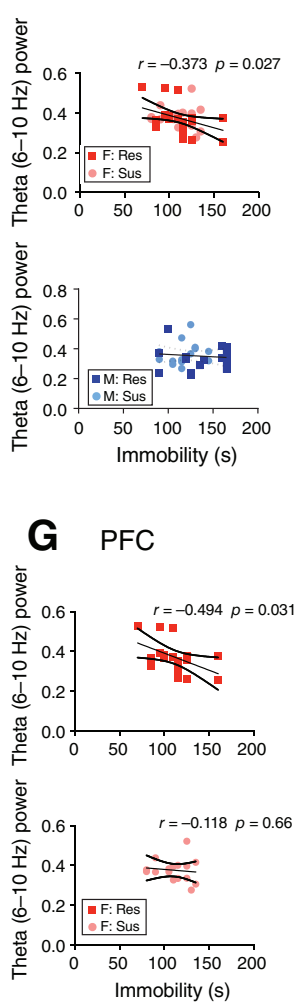

B

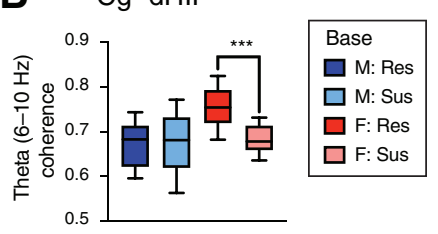

C NAc-dHIP
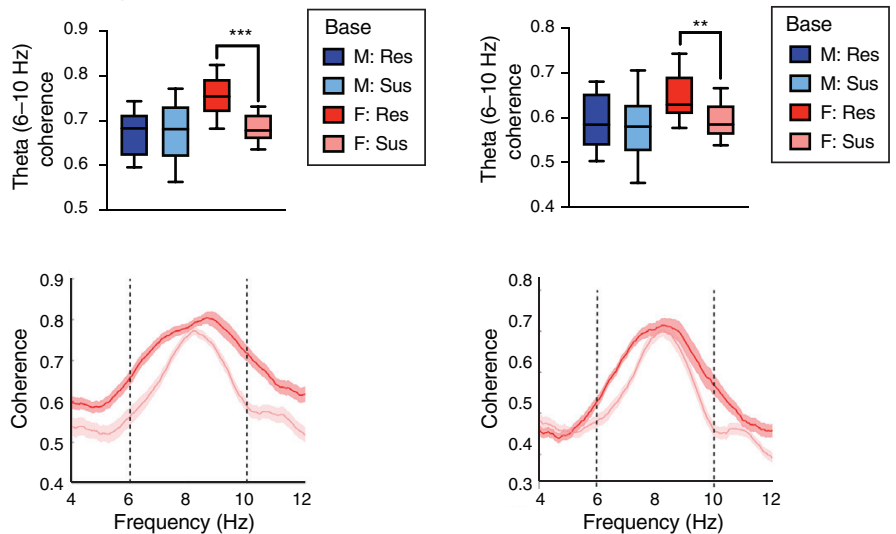

$\mathbf{E}$
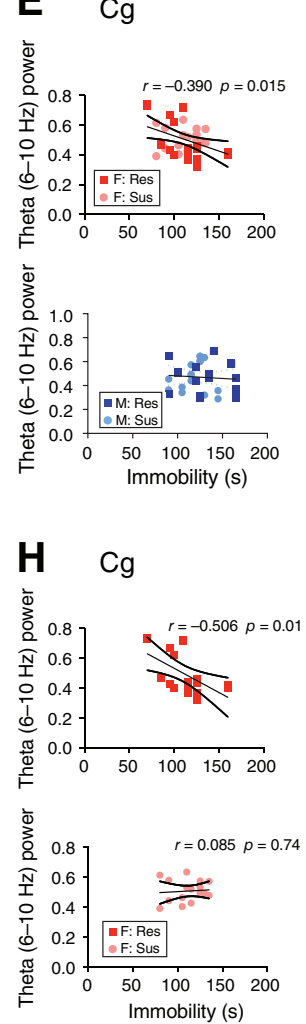

F NAC
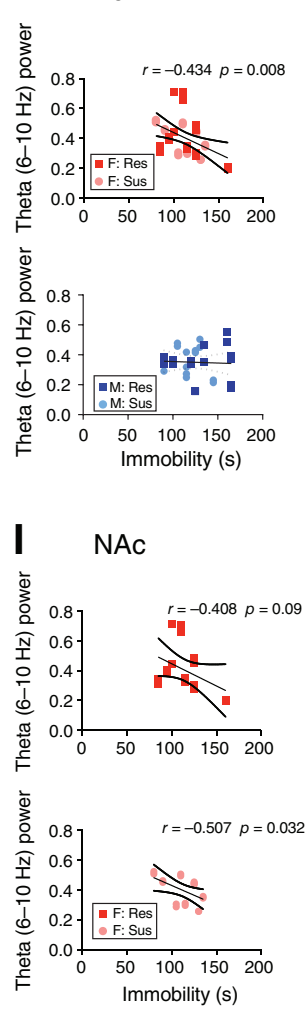

Fig. 2: Baseline sex- and resiliency-dependent differences in theta coherence and correlations between theta power and despair-like behaviour. (A-C) Sex differences in baseline mean theta $(6-10 \mathrm{~Hz})$ frequency coherence (top panel) and theta coherence spectra (bottom panel). At baseline, theta $\left(6-10 \mathrm{~Hz}\right.$ ) coherence in the PFC-dHIP (sex effect, $\left.F_{1,60}=6.374, p=0.014\right), \mathrm{Cg}-\mathrm{dHIP}$ (sex effect, $\left.F_{1,63}=3.850, p=0.05\right)$ and NAc-dHIP (sex effect, $F_{1,61}=15.930, p<0.001$ ) was significantly greater in females subsequently characterized as stress-resilient, than in animals later categorized as stress-susceptible. (D-F) At baseline, theta $(6-10 \mathrm{~Hz})$ power in the PFC, $\mathrm{Cg}$ and NAc was negatively correlated with time spent immobile in the FST in females (top panel), but not in males (bottom panel; linear regression). (G-I) Partial correlations to examine the effect of group revealed significant negative correlations between immobility time in the FST and baseline theta (6-10 $\mathrm{Hz})$ in the PFC and $\mathrm{Cg}$ of resilient females and the NAc of susceptible females. Coherence spectra are presented as normalized data, with jackknife estimates of SEM shown as shaded areas and baseline data for each sex depicted by a black line, unless otherwise indicated. (A-C) A 2-way analysis of variance was used, and quantified coherence is expressed as box-plots with minimum and maximum values. (D-F) Regression data are presented as individual data points with the line of best fit (solid line) \pm SEM (dotted lines). ${ }^{* *} p<0.01,{ }^{* * *} p<0.001$ compared with resilient. $n=$ 8-10 per group, 2 electrodes per region per rat. $\mathrm{Cg}=$ cingulate cortex; dHIP = dorsal hippocampus; FST = forced swim test; NAc = nucleus accumbens; PFC = prefrontal cortex; Res = resilient; SEM = standard error of the mean; Sus = susceptible. 
may act as predictive markers of the stress response. Females categorized as stress-resilient exhibited higher baseline theta $(6-10 \mathrm{~Hz})$ coherence in the dHIP connections (specifically PFC-dHIP [ $p=0.001], \mathrm{Cg}-\mathrm{dHIP}[p<0.001]$ and NAc$\mathrm{dHIP}[p=0.002]$ ) than stress-susceptible females (Figure 2A$\mathrm{C})$; we found no changes in males. Only female rats displayed a significant negative correlation between forced swim test immobility time and theta $(6-10 \mathrm{~Hz})$ power in the PFC $\left(R^{2}=0.139, F_{34}=5.325, p=0.027\right), \operatorname{Cg}\left(R^{2}=0.152, F_{37}=\right.$ $6.461, p=0.015)$ and NAc $\left(R^{2}=0.188, F_{35}=7.894, p=0.008\right.$; Fig. 2D-F), but not in the dHIP. We observed no significant correlations between theta power and sucrose preference. We then performed partial correlations to examine the effect of group in females. We observed a significant negative correlation in the PFC $\left(R^{2}=0.244, F_{17}=5.494, p=0.031\right)$ and $\mathrm{Cg}\left(R^{2}=0.314, F_{18}=8.234, p=0.01\right)$ of resilient females and the NAc $\left(R^{2}=0.257, F_{16}=5.537, p=0.032\right)$ of susceptible females (Figure 2G-I).

\section{Sex-and resiliency-dependent changes in stress-induced oscillatory power}

We next examined the effect of CUS on oscillatory power in each sex. For analysis of stress-susceptible animals, we used the LFP data from the day before their first expression of a depression-like phenotype. For analysis of stress-resilient animals, we used data from the last day of CUS exposure. For all stress-associated analyses, we used theta power at $6-10 \mathrm{~Hz}$ for the PFC, Cg and NAc, and at 7-9 Hz for the dHIP, unless otherwise noted. The theta range for the dHIP was different because it encompasses a unique theta spike that is not present in the other regions.

In the PFC, only stress-susceptible females exhibited increased delta power $(p<0.001)$ and reduced theta power $(p<0.001)$ compared to baseline (Figure 3A). Stress-resilient females displayed increased beta $(p<0.001)$, low gamma $(p=0.001)$ and high gamma power $(p=0.001)$ following CUS; we observed no changes in males (Figure 3B).

In the $\mathrm{Cg}$, CUS enhanced delta power and reduced theta power in stress-susceptible males (delta $p=0.004$; theta $p=0.004$ ) and females (delta $p<0.001$, theta $p<0.001$ ), and more robust theta power reduction in females (Figure $3 C$ ). Further, only stress-resilient female rats exhibited increased Cg high gamma power ( $p=0.001)$ following CUS (Figure 3D).

In the NAc, we observed a stress-induced reduction in theta power in susceptible females only ( $p<0.001$; Figure 3E). As well, we observed increased beta $(p<0.001)$ and low gamma power $(p=0.006)$ exclusively in stress-resilient females (Figure 3F).

In the dHIP, we again observed increased delta power and reduced theta power $(7-9 \mathrm{~Hz})$ in stress-susceptible males (delta $p=0.001$, theta $p=0.007$ ) and females (delta $p=0.001$, theta $p=0.004$ ) following CUS. Conversely, stress-resilient females displayed increased theta power (7-9 Hz; $p<0.001$; Figure 3G). Notably, we found a significant increase in high gamma power in stress-resilient males compared to baseline $(p=0.004)$, and we found a trend of reduced high gamma power in stress-susceptible males (Figure $3 \mathrm{H}$ ).

\section{Sex-and resiliency-dependent changes in stress-induced oscillatory coherence}

We next evaluated stress-induced alterations in inter-regional communication through coherence (Figure 4 and Figure 5). Following CUS, alterations in theta coherence occurred in both sexes. In stress-susceptible female rats, stress decreased theta coherence in the PFC-Cg $(p=0.001)$, PFC-NAc $(p<0.001)$ and Cg-NAc $(p=0.009)$ connections compared to baseline; no changes were evident in stress-resilient females (Figure 4A-C). Conversely, stress-resilient males exhibited increased theta coherence in the PFC-NAc $(p<0.001)$ and Cg-NAc $(p=0.003)$ connections compared to baseline (Figure $4 \mathrm{~B}$ and $\mathrm{C}$ ), and a widespread increase in high gamma coherence (PFC-Cg $p=$ 0.003, PFC-dHIP $p<0.001$, Cg-NAc $p=0.004$, Cg-dHIP $p=$ 0.001, NAc-dHIP $p<0.001$; Figure 5). In the PFC-NAc connection, we observed a trend toward a stress-induced increase in high gamma coherence in stress-resilient males, significantly different from stress-susceptible males ( $p=0.001$; Figure 5B).

\section{Predictive capacity of sex-dependent, stress-induced oscillatory alterations}

We evaluated the time course of oscillatory changes to determine whether early-onset changes could predict the subsequent development of a depression-like phenotype (Figure 6). In stress-susceptible females, theta power reductions preceded delta frequency increases (Figure 6A and Appendix 1, Figure S4A-D); changes occurred first in the PFC, and then in the NAc, $\mathrm{Cg}$ and dHIP (Figure 6A and Appendix 1, Figure S4A-D). We also observed low-frequency power changes in the $\mathrm{Cg}$ and $\mathrm{dHIP}$ in stress-susceptible males, but these occurred earlier and simultaneously (Figure 6B and Appendix 1, Figure S5A-D). Stress-resilient females exhibited increased theta power (7-9 Hz) in the dHIP (Figure 3G), an early adaptive stress response that occurred 2 days after the initiation of CUS (Figure 6A and Appendix 1, Figure S4D). We identified stresssusceptible females by reduced theta coherence in the dHIP connections at baseline (Figure 2A-C), with additional stressinduced reductions in theta coherence in the PFC-Cg, PFCNAc and Cg-NAc connections (Figure 4). These stress-induced reductions in theta coherence developed first in the $\mathrm{PFC}-\mathrm{Cg}$ and the PFC-NAc connections, emerging 12 days after the start of CUS, with the same pattern in the Cg-NAc connection 5 days later (Figure 6A and Appendix 1, Figure S4E-G).

A marker of female resilience was increased gamma power in the PFC, Cg and NAc; we observed no changes in males (Figure 3B, D and F). The low gamma power increase in the PFC preceded that of the NAc; high gamma power was first increased in the $\mathrm{Cg}$, followed by the PFC (Figure 6A and Appendix 1, Fig. S4H-J). In stress-resilient males, increased high gamma power in the dHIP occurred on the last day of CUS (Figure 6B and Appendix 1, Figure S5E). These rats also exhibited a widespread increase in high gamma coherence (Figure 5), first in the dHIP connections and followed by the PFC-Cg and Cg-NAc connections (Figure 6B and Appendix 1, Figure S6A$\mathrm{F})$; increased theta coherence occurred first in the PFC-NAc connection and then in the $\mathrm{Cg}-\mathrm{NAc}$ connection (Figure $6 \mathrm{~B}$ and 


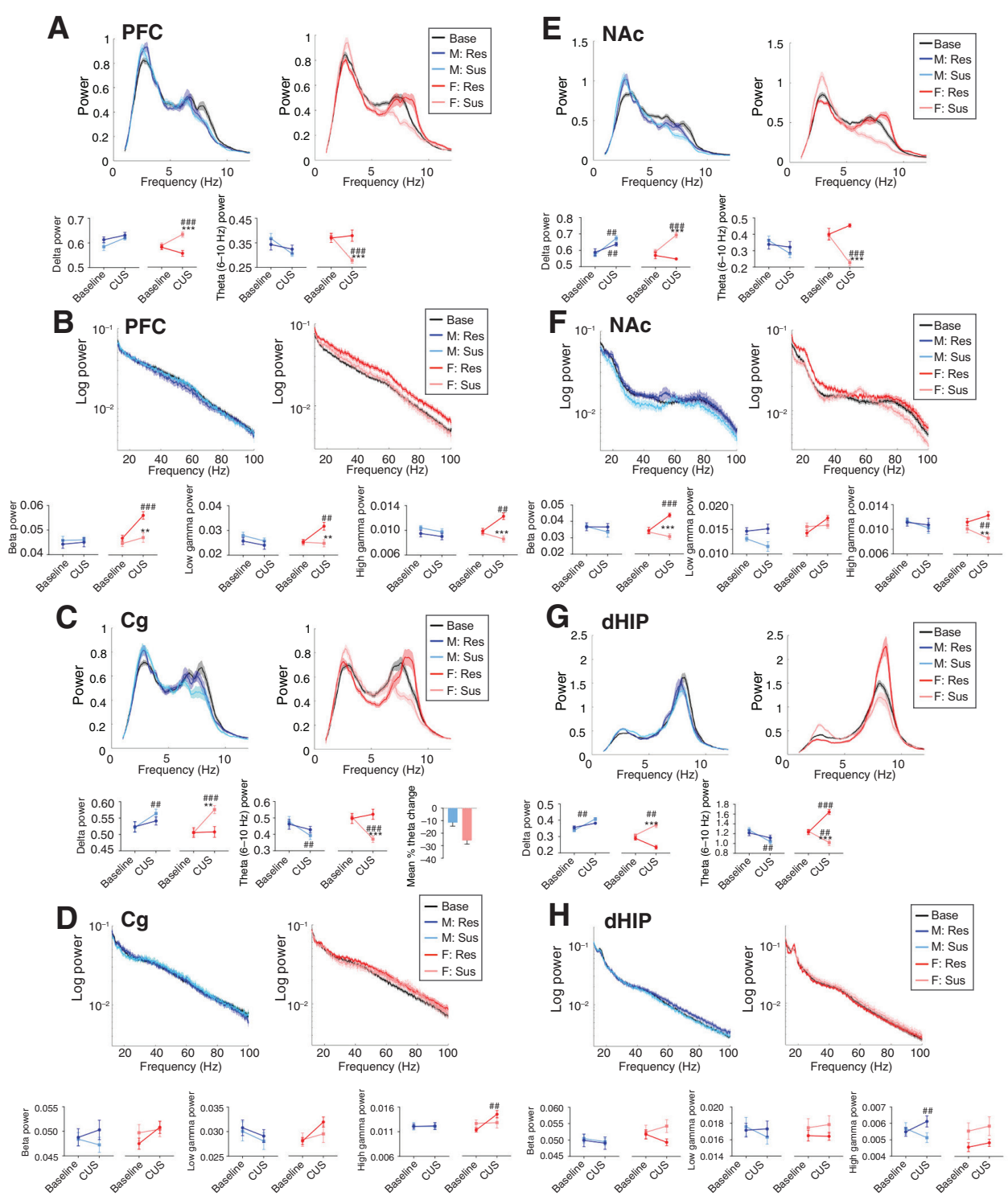

Fig. 3: Sex- and region-dependent alterations in spectral power in response to CUS in susceptible and resilient rats. Normalized low- and highfrequency band power (top panels) and quantification of power spectra at each frequency (bottom panels). (A) In the PFC, susceptible females exhibited increased delta power (time $\times$ resiliency $\times$ sex interaction, $F_{1,56}=7.977, p=0.007$ ), as well as decreased theta $(6-10 \mathrm{~Hz})$ power (time $\times$ resiliency $\times$ sex interaction, $F_{1,58}=4.347, p=0.041$ ). (B) Resilient females displayed increased power in all high-frequency bands in the PFC (time $\times$ resiliency $\times$ sex interaction, beta $F_{1,59}=4.501, p=0.038$; low gamma: $F_{1,61}=4.121, p=0.047$; high gamma: $\left.F_{1,55}=8.410, p=0.005\right)$. (C) In the Cg, susceptible animals of both sexes exhibited increased delta (time $\times$ resiliency interaction, $\left.F_{1,60}=7.116, p=0.001\right)$ and reduced theta $(6-10 \mathrm{~Hz}$ ) power (time $\times$ resiliency $\times$ sex interaction, $F_{1,60}=4.792, p=0.032$ ). This decrease in theta power was more robust in female rats $\left({ }^{* *} p<0.01\right.$ compared with male rats, Student $t$ test, bottom right panel). (D) In the $\mathrm{Cg}$, we observed increased high gamma power in resilient females (time $\times$ sex interaction, $F_{1.57}=9.583, p=0.003$ ). $(E)$ In the NAc, susceptible females showed a reduction in theta $(6-10 \mathrm{~Hz})$ power (time $\times$ resiliency $\times$ sex interaction, $\left.F_{1,57}=11.176, p=0.001\right)$. $(F)$ In the NAc, resilient females displayed an increase in beta (time $\times$ resiliency $\times$ sex interaction, $F_{1,60}=$ 4.039, $p=0.049$ ) and low gamma power (time $\times$ resiliency interaction, $F_{1,59}=8.93, p=0.004$ and time $\times$ sex interaction, $F_{1,59}=4.775, p=0.033$ ), and susceptible females had reduced high gamma power (time $\times$ resiliency interaction, $F_{1.58}=3.989, p=0.05$ and time $\times$ sex interaction, $F_{1.58}=$ 4.587, $p=0.036$ ). (G) In the dHIP, both susceptible males and females had increased delta power (time $\times$ resiliency $\times$ sex interaction, $F_{1,51}=4.254$, $p=0.044)$ and reduced theta $(7-9 \mathrm{~Hz})$ power (time $\times$ resiliency $\times$ sex interaction, $F_{1,53}=4.681, p=0.035$ ), and theta power was increased in resilient females. $(H)$ In the dHIP, high gamma power was increased in resilient males (time $\times$ resiliency $\times$ sex interaction, $F_{1,57}=9.035, p=0.004$ ). Power spectra are presented as normalized data with jackknife estimates of SEM shown as shaded areas and with baseline data for each sex depicted by a black line. The quantified power spectra are presented as mean \pm SEM. A 3-way repeated-measures analysis of variance was used, unless otherwise indicated. ${ }^{* *} p<0.01,{ }^{* * *} p<0.001$ compared with resilient animals; ${ }^{* \#} p<0.01,{ }^{\# \#} p<0.001$ compared with baseline. $n=8-10$ per group, 2 electrodes per region per rat. $\mathrm{Cg}=$ cingulate cortex; CUS = chronic unpredictable stress; $\mathrm{dHIP}=$ dorsal hippocampus; EPM = elevated plus maze; $\mathrm{NAc}=$ nucleus accumbens; $\mathrm{PFC}=$ prefrontal cortex; Res = resilient; $\mathrm{SEM}=$ standard error of the mean; Sus = susceptible. 


\section{A $\quad \mathrm{PFC}-\mathrm{Cg}$}
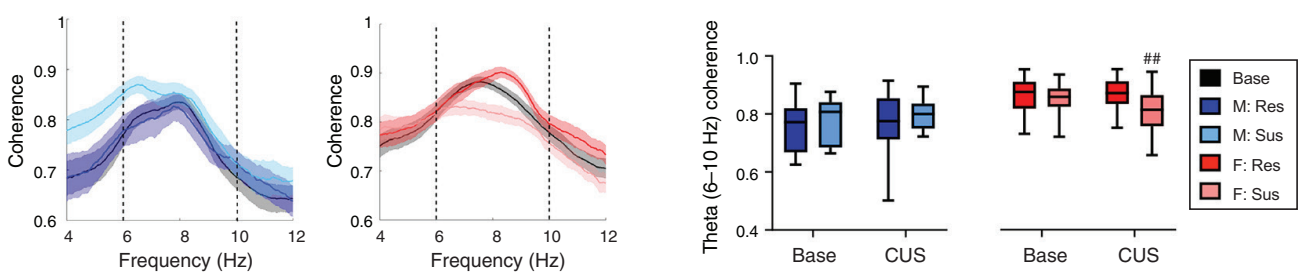

B PFC-NAC
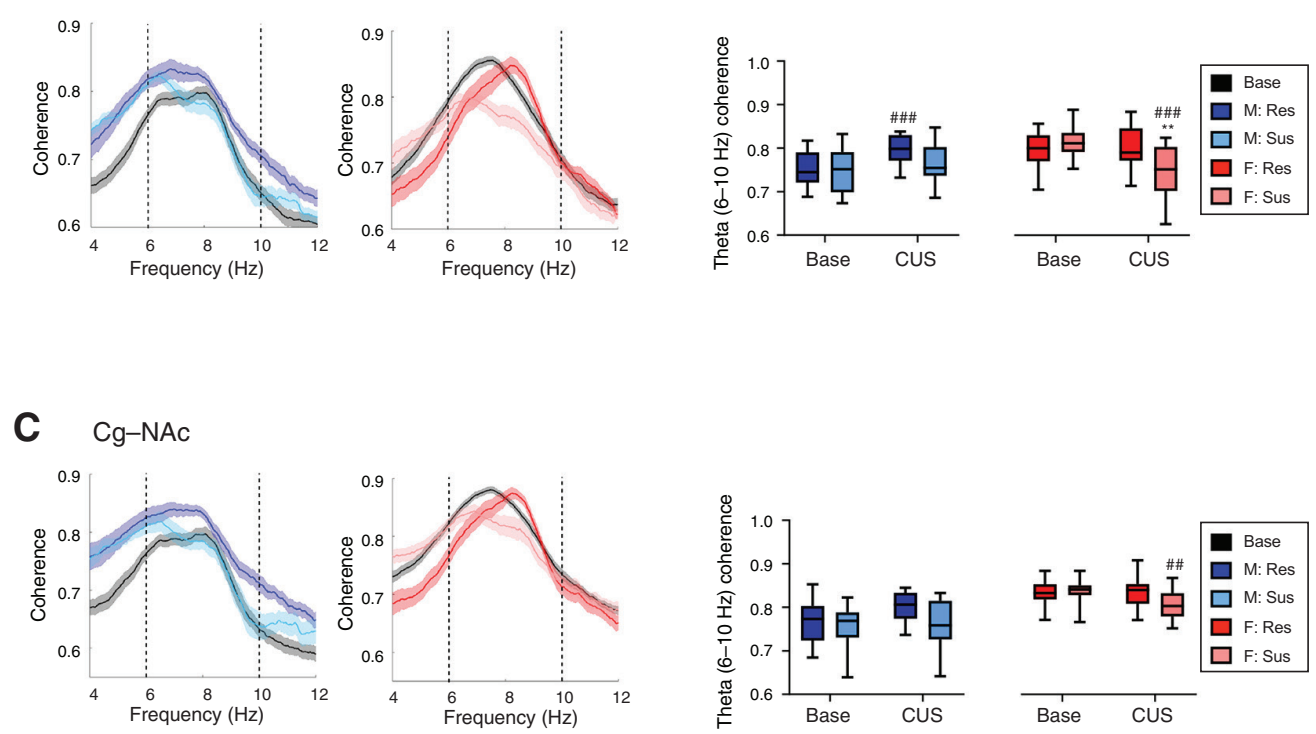

Fig. 4: Sex-dependent alterations in theta frequency coherence in response to CUS in susceptible and resilient rats. Theta coherence spectra (left panel) and sex-dependent CUS-induced changes in mean theta $(6-10 \mathrm{~Hz})$ frequency coherence (right panel). (A) In the PFC-Cg, susceptible females displayed reduced theta $(6-10 \mathrm{~Hz})$ coherence following CUS exposure (time $\times$ sex interaction, $F_{1,53}=7.094, p=0.01$ ). $(B, C)$ Stress induced decreased and increased theta $(6-10 \mathrm{~Hz})$ coherence in susceptible females and resilient males, respectively, in the PFC-NAc (time $\times$ resiliency interaction, $F_{1,60}=27.482, p<0.001$; and time $\times$ sex interaction, $F_{1,60}=33.511, p<0.0001$ ) and the Cg-NAc (time $\times$ resiliency interaction, $F_{1,57}=5.508, p=0.022$; and time $\times$ sex interaction, $F_{1,57}=9.836, p=0.003$ ). Coherence spectra are presented as normalized data with jackknife estimates of SEM shown as shaded areas and with baseline data for each sex depicted by a black line, unless otherwise indicated. Quantified coherence is expressed as box-plots with minimum and maximum values. A 3-way repeated-measures

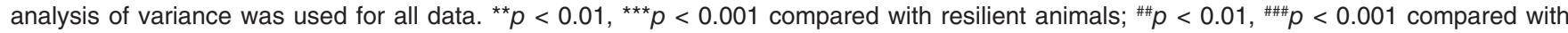
baseline. $n=8-10$ per group, 2 electrodes per region per rat. $\mathrm{Cg}=$ cingulate cortex; $\mathrm{CUS}=$ chronic unpredictable stress; $\mathrm{dHIP}=$ dorsal hippocampus; NAc = nucleus accumbens; PFC = prefrontal cortex; Res = resilient; SEM = standard error of the mean; Sus = susceptible.

Appendix 1, Figure S6G and H). These results illustrate a sexspecific and time-dependent recruitment of brain regions and pathways with stress exposure, indicated by frequency-specific changes in the function of neural oscillatory systems.

\section{Discussion}

This study evaluated sex differences in oscillatory patterns associated with exposure to CUS in rats and the potential of these patterns to serve as early indices of depression-like behaviour. We demonstrated that male and female animals exhibited region-dependent differences in stress-induced neurophysiological patterns, and that specific patterns were predictive of susceptibility. Further, resiliency was not associated simply with a lack of susceptibility signatures but manifested with distinct sex-specific innate and stress-induced oscillatory changes. Overall, susceptibility or resiliency to repeated stress exposure was associated with a temporal recruitment of circuits, culminating in system-wide changes that coincided with the onset of depression-like behaviour.

Consistent with clinical ${ }^{4,5}$ and preclinical ${ }^{6,7,51}$ reports, female rats were more sensitive to stress; they required a shorter duration of stress exposure to induce a depressionlike phenotype than males. Stress-susceptible females were 
A $\mathrm{PFC}-\mathrm{Cg}$
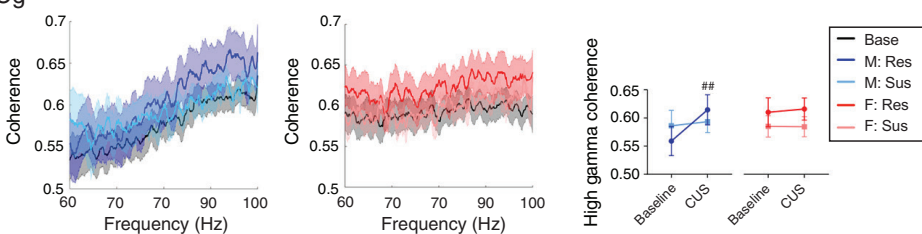

B PFC-NAC
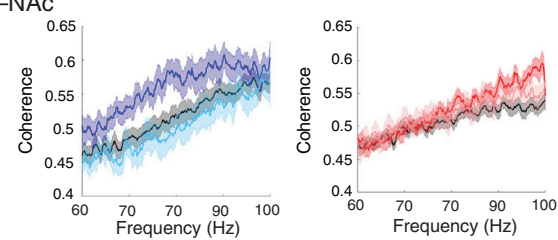

C PFC-dHIP
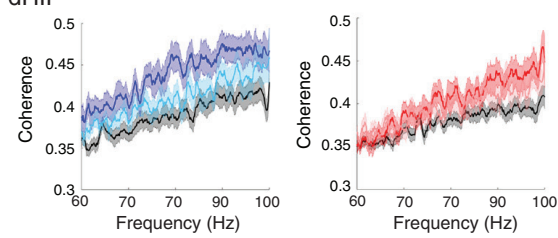

D Cg-NAc
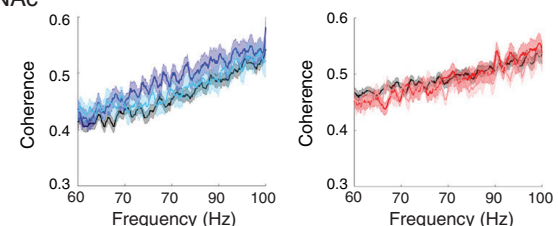

E $\mathrm{Cg}-\mathrm{dHIP}$
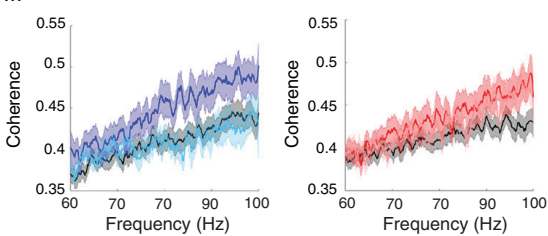

F NAc-dHIP
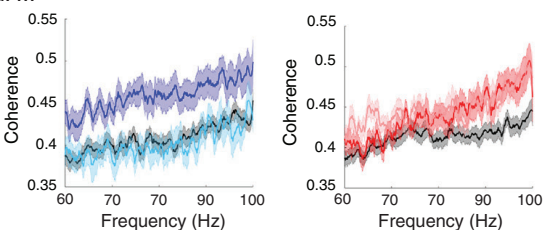
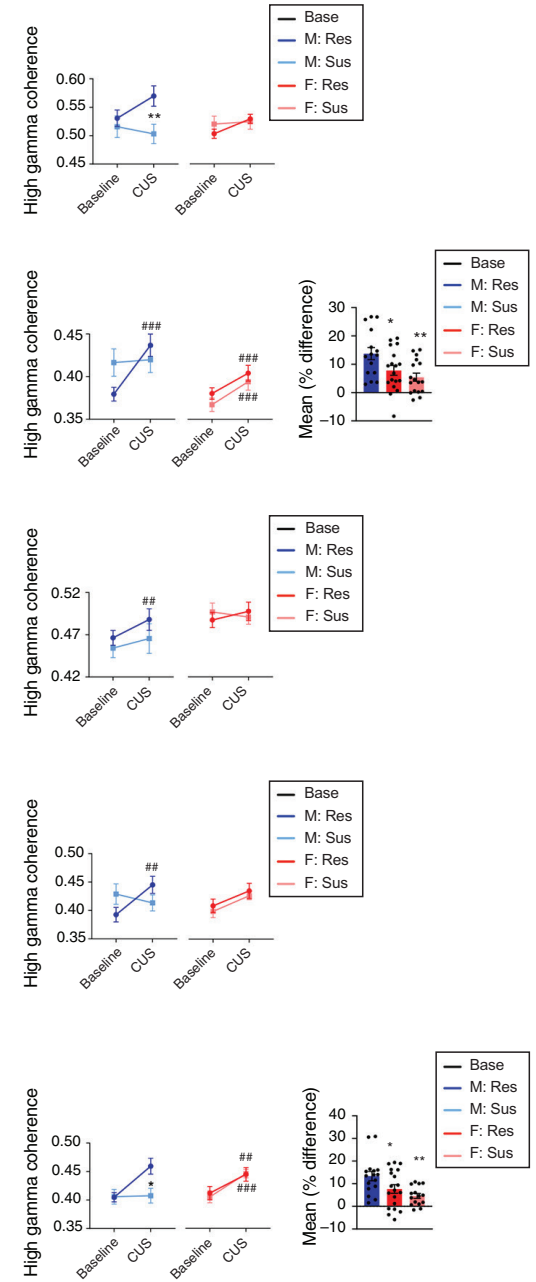

Fig. 5: Sex-dependent alterations in high gamma frequency coherence following CUS exposure in susceptible and resilient rats. High gamma frequency coherence spectra for males (left panel) and females (centre panel) and mean coherence values (right panel). (A) In the PFC-Cg, only resilient males displayed increased high gamma coherence (time $\times$ resiliency interaction, $F_{1,60}=7.673, p=0.007$ and sex $\times$ resiliency interaction, $\left.F_{1,60}=3.816, p=0.05\right)$. (B) In the PFC-NAc, resilient males exhibited an increasing trend of high gamma coherence from baseline, and this was significantly different from susceptible males (time $\times$ resiliency interaction, $F_{1.55}=5.771, p=0.02$ ). (C) Resilient males and females, as well as susceptible females, had increased high gamma coherence in the PFC-dHIP (time $\times$ resiliency $\times$ sex interaction, $F_{1,56}=11.973, p=0.001$ ), with greater effects observed in the male rats $\left({ }^{*} p<0.05\right.$ and ${ }^{* *} p<0.01$ compared to male rats, Student $t$ test, far right panel). (D, E) Only resilient males exhibited increased high gamma coherence in the Cg-NAc (time $\times$ resiliency interaction, $F_{1,53}=9.374, p=0.003$ and main effect of sex, $F_{1,53}=8.448, p=0.005$ ) and the $\mathrm{Cg}-\mathrm{dHIP}$ (time $\times$ resiliency $\times$ sex interaction, $F_{1,56}=4.729, p=0.034$ ). (F) We observed increased high gamma coherence in resilient males and females, as well as in susceptible females, in the NAc-dHIP (time $\times$ resiliency $\times$ sex interaction, $F_{1,57}=8.145, p=$ 0.006), but it was more robust in resilient males ( ${ }^{*} p<0.05$ and ${ }^{* *} p<0.01$ compared with male rats, Student $t$ test, far right panel). Coherence spectra are presented as normalized data with jackknife estimates of SEM shown as shaded areas and with baseline data for each sex depicted by a black line. Quantified coherence is presented as mean \pm SEM. A 3-way repeated-measures analysis of variance was used, unless otherwise indicated. ${ }^{* *} p<0.01,{ }^{* * *} p<0.001$ compared with resilient animals; ${ }^{* \#} p<0.01$, ${ }^{\# \#} p<0.001$ compared with baseline. $n=8-10$ per group, 2 electrodes per region per rat. $\mathrm{Cg}=$ cingulate cortex; $\mathrm{CUS}=$ chronic unpredictable stress; $\mathrm{dHIP}=$ dorsal hippocampus; NAc $=$ nucleus accumbens; PFC = prefrontal cortex; Res = resilient; SEM = standard error of the mean; Sus = susceptible. 
A
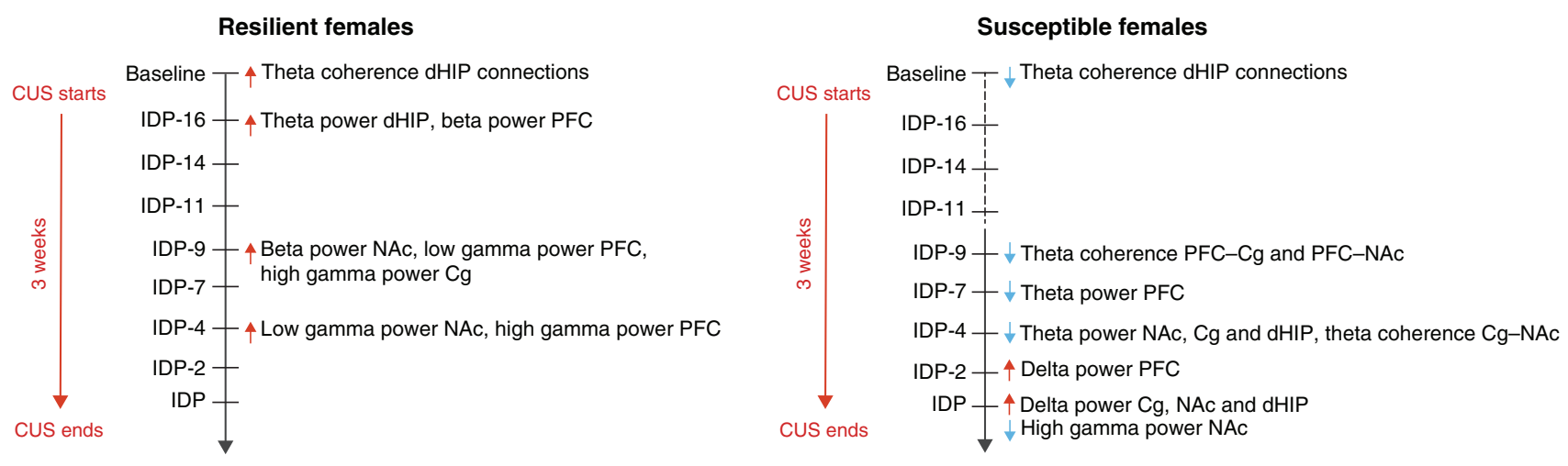

B

Resilient males

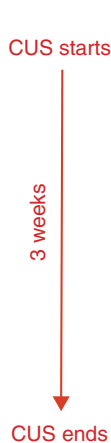$$
\text { Base }
$$
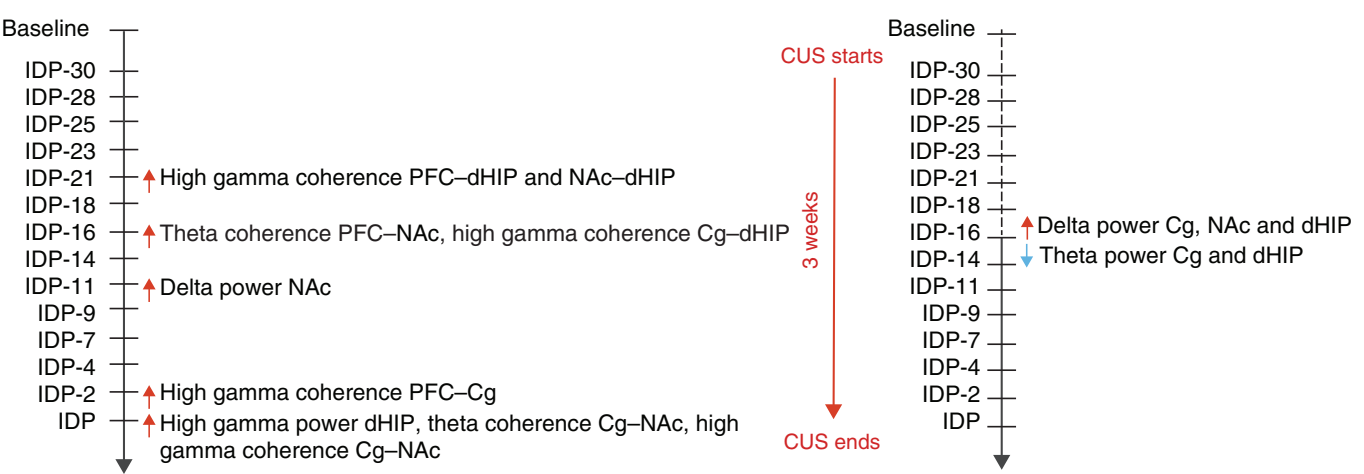

Fig. 6: Timeline of sex-dependent early oscillatory alterations predictive of resilience or susceptibility to chronic stress exposure in rats. (A) At baseline, resilient females could be distinguished from susceptible females due to their greater theta $(6-10 \mathrm{~Hz})$ coherence in the $\mathrm{dHIP}$ connections. Resilient females also exhibited an early theta $(7-9 \mathrm{~Hz})$ frequency power increase in the dHIP, as well as increased highfrequency power changes. In contrast, susceptible females displayed global increased and decreased delta and theta power, respectively, in addition to reduced theta $(6-10 \mathrm{~Hz})$ coherence in the PFC-Cg-NAc circuit. (B) Resilient males were predominantly characterized by a widespread elevation in high gamma coherence, which was first observed in the NAc-dHIP and the PFC-dHIP after 10 days of stress exposure. This increased high gamma coherence then sequentially occurred in all other connections, with the exception of the PFC-NAc, suggesting that male resilience is dependent on the temporal recruitment of circuits that confer protection against chronic stress exposure. Conversely, susceptible males were largely marked by increased delta power and reduced theta power in the $\mathrm{Cg}$ and dHIP. Because distinct patterns of susceptibility were minimal, it may be that markers of resilience are more critical in male stress responses, rather than markers of susceptibility. The timeline highlights the temporal dynamic of the adaptive changes in response to stress and that there are specific neurophysiological responses that confer resilience, thereby preventing the onset of those oscillatory changes associated with susceptibility. $\mathrm{Cg}=$ cingulate cortex; $\mathrm{dHIP}=$ dorsal hippocampus; $\mathrm{NAC}=$ nucleus accumbens; $\mathrm{PFC}=$ prefrontal cortex.

distinguishable from stress-resilient females through differences in low-frequency activity. Specifically, baseline theta coherence in the dHIP connections of female stress-resilient animals was greater than that in stress-susceptible animals. Further, whereas stress-susceptible females exhibited a system-wide increase in delta power concomitant with decreased theta power, stress-resilient females exhibited a significant upregulation of theta power selectively in the dHIP. Low-frequency power changes were also apparent in males: stress-susceptible animals showed a stress-induced increase in delta power and a decrease in theta power - effects lim- ited to the $\mathrm{Cg}$ and $\mathrm{dHIP}$, and with no opposing patterns in stress-resilient males.

The interpretation of these findings is complex, because sex differences in the function of neural systems in MDD have been poorly characterized. ${ }^{50}$ As well, clinical reports examining lowfrequency activity in MDD show inconsistent findings, possibly because sex was not used as an inclusive experimental variable. In support of our findings, clinical EEG studies showed increased global or frontal delta activity in people with MDD ${ }^{52-54}$ and in women with menopausal depression. ${ }^{55}$ Increased temporal theta and alpha power have also been associated with 
improved mood, ${ }^{56}$ whereas a global cortical reduction in theta and alpha power has been reported in MDD. ${ }^{57}$ This reduced theta power in the occipitoparietal regions of patients with MDD may reflect impaired affect regulation and functional connectivity, ${ }^{58,59}$ and indeed, cortical theta hypoconnectivity in MDD has been documented. ${ }^{54,60}$ In preclinical studies, a genetic mouse model of depression also showed reduced baseline theta oscillatory power in the PFC, correlated with greater freezing time in the tail suspension test. ${ }^{28}$ These findings demonstrating a link between reduced theta connectivity and MDD complemented our own results showing pathway-specific and temporal reductions in theta power in stress-susceptible rats, as well as increases in innate and stress-induced theta coherence in stress-resilient female and male rats, respectively. These sex differences in brain function in response to stress emphasize that the manifestation of depression symptoms in males and females may be mediated by distinct mechanisms.

Sex-specific alterations in high-frequency oscillations were linked to stress susceptibility, and more so to stress resilience. Compared to baseline, stress-susceptible females displayed reduced high gamma power in the NAc; stress-resilient females displayed increased gamma power in the PFC, Cg and NAc; and stress-resilient males showed widespread increases in high gamma coherence. A role for gamma oscillations in MDD and antidepressant responsiveness has been documented, although to our knowledge sex differences in these measures have not been evaluated. Individuals with MDD exhibited lower resting gamma activity in the rostral Cg compared to healthy individuals, ${ }^{27}$ and previous preclinical findings were consistent with our own, showing that reduced gamma power in the PFC and NAc was associated with a depression-like phenotype. ${ }^{28,29}$ Increased resting gamma power in MDD (particularly in the PFC) in response to repetitive transcranial magnetic stimulation has been linked to successful remission, ${ }^{30-32}$ findings supported by rodent studies showing that ketamine treatment increased gamma oscillatory activity in the PFC, NAc, amygdala, hippocampus and thalamus. ${ }^{39,61,62}$ Similarly, deep brain stimulationinduced normalization of suppressed high gamma coherence in the ventral tegmental area of Flinders rats (a model system useful for the study of MDD) was correlated with improved immobility measures in the forced swim test. ${ }^{63}$

Our findings provide much needed insights into sex-specific, regional and temporal changes that occur in response to stress, emphasizing distinct oscillatory markers linked to resilience and vulnerability. At baseline, females had greater theta coherence across the dHIP connections, successfully distinguishing between animals that were subsequently labelled as stress-resilient or stress-susceptible. Indeed, 48 hours after the onset of CUS, female stress resilience was accompanied by increased dHIP theta power, with opposite effects in stress-susceptible females. Notably, the aforementioned increase in gamma power displayed by stress-resilient females developed after the change in dHIP theta frequency was established. Taken together, these findings suggest that the dHIP may function as a fulcrum for female stress resilience, in which distinct low-frequency alterations may be necessary to initiate events that trigger increases in highfrequency power in the PFC, $\mathrm{Cg}$ and NAc. In males, our findings and others suggest that a rapid and progressive stress-induced increase in high gamma coherence may contribute to stress resilience. Once again, stress resilience was associated with the temporal recruitment of almost all of the examined connections in the putative depression network, beginning in the dHIP connections. Thus, although the neurophysiological mechanisms may differ, we hypothesize that the temporal recruitment of regions, potentially initiated by the dHIP, confers protection against stress.

Given the temporal dynamics of the observed adaptive changes associated with stress, we posit that the neurophysiological responses that confer stress resilience play a key role in preventing the onset or minimizing the functional impact of oscillatory changes associated with stress susceptibility, and that the nature of these changes are sex-specific. The importance of these resilience markers was perhaps most obvious in male rats, where resilient animals showed robust widespread elevations in high gamma coherence, but the only clear susceptibility marker was a stress-induced increase in delta power and a reduction in theta power in the $\mathrm{Cg}$ and $\mathrm{dHIP}$. Conversely, in female rats the PFC appeared to be highly vulnerable to stress, being the first structure to exhibit stress-induced changes in low-frequency power in susceptible animals - changes soon followed by low-frequency alterations throughout the network. However, the appearance of many of these discrete susceptibility markers occurred several days before the initial expression of depression-like symptoms. This signifies that in females, oscillatory changes in discrete pathways may be insufficient to induce depression-like symptoms; system-wide changes may be necessary for the behavioural expression of the depression-like phenotype.

\section{Limitations}

This study was designed to determine whether sex-specific differences in neural oscillations could be used as biological markers for stress vulnerability, but the methodology we employed could not establish causation of stress resilience or susceptibility using the electrophysiological patterns. However, the small margin of error within each group suggests an association between the 2 variables. Further, high-frequency deep brain stimulation in patients with treatment-resistant depression produced a sustained antidepressant response, ${ }^{9,64}$ highlighting the link between oscillatory activity and depression. Future studies may mimic key oscillatory markers observed in stress-susceptible animals using deep brain stimulation to validate their ability to induce depression-like behaviours.

Another limitation was the use of monopolar electrodes for LFP recordings. Monopolar electrode recordings are subject to volume conductance, so the signals detected may not necessarily be generated from the recording region. ${ }^{65}$ Although this may be less of a concern for the dHIP data, the NAc data could be subject to volume conductance from the adjacent piriform cortex. However, because this study was the first of its kind, maximizing the number of bilateral regions evaluated was a priority to examine potential data asymmetries. Thus, the number of regions that could be evaluated simultaneously was a technical limitation. Because we did not find evidence of asymmetries, 
future studies using bipolar electrodes will be required to fully elucidate the role of each brain region in the observed effects.

As a result of the within-subjects design of the study, control animals subjected only to the weekly behavioural testing were not included. As was the case with this experiment, the forced swim test can be used as a stressor to induce depression-like behaviours in rodents. ${ }^{66}$ As such, there may have been a confounding effect of weekly exposure to the forced swim test on circuit function that was not apparent in this study because of the lack of control animals. It should also be noted that although CUS exposure was stopped when half of the animals exhibited a depression-like phenotype, the stress-resilient animals would have also eventually succumbed to the stressors to develop depression-like behaviours. Although we surmise that their oscillatory signatures would have changed, we cannot say for certain that they would have mimicked a stresssusceptible phenotype or in which regions these alterations would have occurred. In line with this, we did not examine the adaptability of the circuit with prolonged stress exposure and recovery of the system following the cessation of chronic stress; these should be the focus of future studies.

\section{Conclusion}

Our findings demonstrated that stress-induced changes occur in both low- and high-frequency neurophysiological responses that are unique to each sex. In addition, stress exposure induced the sex-dependent temporal recruitment of circuits that were linked to susceptibility or resilience to the development of depression-like behaviours. Given the difficulty in treating depression, identification of people at risk (e.g., those with a personal or family history of depression, postpartum depression, or exposure to a traumatic event) could lead to more precise monitoring and interventions as necessary, at a stage before onset of depression, with significant potential for increased treatment efficacy and drug responsiveness. Conversely, because oscillatory markers of resiliency in both sexes occurred early in stress exposure, we posit that the presence or absence of innate or stress-induced resilience signatures is a critical determinant of an animal's subsequent stress response. Finally, our results highlight that stress influences neural oscillations in a sex-specific manner; the inclusion of sex as an experimental factor in future clinical and preclinical studies should be made a priority if we are to fully elucidate the neuropathology of MDD and develop more effective treatments.

Affiliations: From the department of Molecular and Cellular Biology, University of Guelph, Guelph, Ont., Canada (Thériault, Manduca, Perreault) and the Collaborative Neuroscience Program, University of Guelph, Guelph, Ont., Canada (Thériault, Perreault).

Funding: This research was supported by the Canadian Institutes of Health Research (grant number 450186 to MLP) and a Vanier Canada Graduate Scholarship (RKT).

Competing interests: None declared.

Contributors: M. Perreault designed the study. R.-K. Thériault and J. Manduca acquired the data, which R.-K. Thériault and M. Perrault analyzed. R.-K. Thériault wrote the article, which all authors reviewed. All authors approved the final version to be published and can certify that no other individuals not listed as authors have made substantial contributions to the paper.

Content licence: This is an Open Access article distributed in accordance with the terms of the Creative Commons Attribution (CC BYNC-ND 4.0) licence, which permits use, distribution and reproduction in any medium, provided that the original publication is properly cited, the use is non-commercial (i.e. research or educational use), and no modifications or adaptations are made. See: https://creativecommons.org/licenses/by-nc-nd/4.0/

\section{References}

1. McKenna MT, Michaud CM, Murray CJ, et al. Assessing the burden of disease in the United States using disability-adjusted life years. Am J Prev Med 2005;28:415-23.

2. Addis ME. Why is depression more prevalent in women? Clin Psychol Sci Pract 2008;15:153-68.

3. LaPlant Q, Chakravaty S, Vialou V, et al. Role of nuclear factor $\mathrm{kB}$ in ovarian hormone-mediated stress hypersensitivity in female mice. Biol Psychiatry 2009;65:874-80.

4. Li SH, Graham BM. Why are women so vulnerable to anxiety, trauma-related and stress-related disorders? The potential role of sex hormones. Lancet Psychiatry 2017;4:73-82.

5. Hodes GE. Sex, stress, and epigenetics: regulation of behaviour in animal models of mood disorders. Biol Sex Differ 2013;4:1.

6. Pfau ML, Russo SJ. Peripheral and central mechanisms of stress resilience. Neurobiol Stress 2015;1:66-79.

7. Rincon-Cortes M, Grace AA. Sex-dependent effects of stress on immobility behaviour and VTA dopamine neuron activity: modulation by ketamine. Int J Neuropsychopharmacol 2017;20:823-32.

8. Shors TJ, Chua C, Falduto J. Sex differences and opposites effects of stress on dendritic spine density in the male versus female hippocampus. J Neurosci 2001;21:6292-97.

9. Smart OL, Tiruvadi VR, Mayberg H. Multimodal approaches to define network oscillations in depression. Biol Psychiatry 2015;77:1061-70.

10. Baskaran A, Farzan F, Milev R, et al. The comparative effectiveness of electroencephalographic indices in predicting response to escitalopram therapy in depression: a pilot study. J Affect Disord 2018;227:542-9.

11. Allen JJB, Urry HL, Hitt SK, et al. The stability of resting frontal electroencephalographic asymmetry in depression. Psychophysiology 2004;41:269-80.

12. Henriques JB, Davidson RJ. Regional brain electrical asymmetries discriminate between previously depressed and healthy control subjects. J Abnorm Psychol 1990;99:22-31.

13. Domschke K, Zwanzger P, Rehbein MA. al. Magnetoencephalographic correlates of emotional processing in major depression before and after pharmacological treatment. Int J Neuropsychopharmacol 2016;19:1-9.

14. Gotlib IH, Ranganath C, Rosenfeld JP. Frontal EEG alpha asymmetry, depression, and cognitive functioning. Cogn Emotion 1998;12:449-78.

15. Coan JA, Allen JJB. Frontal EEG asymmetry and the behavioral activation and inhibition systems. Psychophysiology 2003;40:106-14.

16. Jaworska N, Blier P, Fusee W, et al. Alpha power, alpha asymmetry and anterior cingulate cortex activity in depressed males and females. J Psychiatr Res 2012;46:1483-91.

17. Korb AS, Hunter AM, Cook IA, et al. Rosteral anterior cingulate cortex theta current density and response to antidepressants and placebo in major depression. Clin Neurophysiol 2009;120:1313-9.

18. Saletu B, Anderer P, Saletu-Zyhlarz GM. EEG topography and tomography (LORETA) in diagnosis and pharmacotherapy of depression. Clin EEG Neurosci 2010;41:203-10.

19. Mitchell DJ, McNaughton N, Flanagan D, et al. Frontal-midline theta from the perspective of hippocampal "theta." Prog Neurobiol 2008;86:156-85.

20. Mulert C, Juckel G, Brunmeier M, et al. Rostral anterior cingulate cortex activity in the theta band predicts response to antidepressive medication. Clin EEG Neurosci 2007;38:78-81.

21. Mientus S, Gallinat J, Wuebben Y, et al. Cortical hypoactivation during resting EEG in schizophrenics but not in depressives and schizotypal subjects as revealed by low resolution electromagnetic tomography (LORETA). Psychiatry Res 2002;116:95-111. 
22. Lubar JF, Congedo M, Askew J. Low-resolution electromagnetic tomography (LORETA) of cerebral activity in chronic depressive disorder. Int J Psychophysiol 2003;49:175-85.

23. Pizzagalli DA, Nitschke JB, Oakes TR, et al. Brain electrical tomography in depression: the important of symptom severity, anxiety, and melancholic features. Biol Psychiatry 2002;52:73-85.

24. Spronk D, Arns M, Barnett K, et al. An investigation of EEG, genetic and cognitive markers of treatment response to antidepressant medication in patients with major depressive disorder: a pilot study. J Affect Disord 2011;128:41-8.

25. Arns M. First EEG results of the iSPOT study in depression: EEG alpha asymmetry as a gender specific predictor of SSRI treatment outcome. Brain Stimul 2015;8:337.

26. Broadway JM, Holtzheimer PE, Hilimire MR, et al. Frontal theta cordance predicts 6-month antidepressant response to subcallosal cingulate deep brain stimulation for treatment-resistant depression: a pilot study. Neuropsychopharmacology 2012;37:1764-72.

27. Pizzagalli DA, Peccoralo LA, Davidson RJ, et al. Resting anterior cingulate activity and abnormal responses to errors in subjects with elevated depressive symptoms: a 128-channel EEG study. Hum Brain Mapp 2006;27:185-201.

28. Sauer JF, Struber M, Bartos M. Impaired fast-spiking interneuron function in a genetic mouse model of depression. eLife 2015, 4:e04979.

29. Voget M, Rummel J, Avchalumov Y, et al. Altered local field potential activity and serotonergic neurotransmission are further characteristics of the Flinders sensitive line rat model of depression. Behav Brain Res 2015;15:299-305.

30. Noda Y, Zomorrodi R, Saeki T, et al. Resting-state EEG gamma power and theta-gamma coupling enhancement following highfrequency left dorsolateral prefrontal rTMS in patients with depression. Clin Neurophysiol 2017;128:424-32.

31. Bailey NW, Hoy KE, Rogasch NC, et al. Responders to rTMS for depression show increased fronto-midline theta and theta connectivity compared to non-responders. Brain Stimul 2018;11:190-203.

32. Pathak Y, Salami O, Baillet S, et al. Longitudinal changes in depressive circuitry in response to neuromodulation therapy. Front Neural Circuits 2016;10:50.

33. Stewart JL, Bismark AW, Towers DN, et al. Resting frontal EEG asymmetry an endophenotype for depression risk: sex-specific patterns of frontal brain asymmetry. J Abnorm Psychol 2010;119:502-12.

34. Bruder GE, Stewart JW, Tenke CE, et al. Electroencephalographic and perceptual asymmetry differences between responders and nonresponders to an SSRI antidepressant. Biol Psychiatry 2001; 49:416-25.

35. Jesulola E, Sharpley CF, Agnew LL. The effects of gender and depression severity on the association between alpha asymmetry and depression across four brain regions. Behav Brain Res 2017;321:232-9.

36. Nusslock R, Shackman AJ, McMenamin BW, et al. Comorbid anxiety moderates the relationship between depression history and prefrontal EEG asymmetry. Psychophysiology 2018;55:e12953.

37. Jacobs GD, Snyder D. Frontal brain asymmetry predicts affective style in men. Behav Neurosci 1996;110:3-6.

38. Stewart JL, Coan JA, Towers DN, et al. Frontal EEG asymmetry during emotional challenge differentiates individuals with and without lifetime major depressive disorder. J Affect Disord 2011;129:167-74.

39. Manduca JD, Thériault RK, Williams OOF, et al. Transient dosedependent effects of ketamine on neuronal oscillatory activity in Wistar-Kyoto rats. Neuroscience 2020;441:161-75.

40. Dominuges K, Lima FB, Linder AE, et al. Sexually dimorphic responses of rats to fluoxetine in the forced swimming test are unrelated to the function of the serotonin transporter in the brain. Synapse 2020;74:e22130.

41. Mezdari TJ, Batista GM, Portes AC, et al. Repeated rat-forced swim test: reducing the number of animals to evaluate gradual effects of antidepressants. J Neurosci Methods 2011;195:200-5.

42. Possamai F, dos Santos J, Walber T, et al. Influence of enrichment on behavioural and neurogenic effects of antidepressants in Wistar rats submitted to repeated forced swim test. Prog Neuropsychopharmacol Biol Psychiatry 2015;58:15-21.

43. Hasbi A, Nguyen T, Rahal H, et al. Sex difference in dopamine D1D2 receptor complex expression and signaling affects depressionand anxiety-like behaviours. Biol Sex Differ 2020;11:8.

44. Willner P. The chronic mild stress (CMS) model of depression: history, evaluation and usage. Neurobiol Stress 2016;6:78-93.
45. Shen MY, Perreault ML, Bambico FR, et al. Rapid anti-depressant and anxiolytic actions following dopamine D1-D2 receptor heteromer inactivation. Eur Neuropsychopharmacol 2015;25:2437-48.

46. Ho YC, Lin TB, Hsieh MC, et al. Periaqueductal gray glutamatergic transmission governs chronic stress-induced depression. Neuropsychopharmacology 2018;43:302-12.

47. Hutchinson KM, McLaughlin KJ, Wright RL, et al. Environmental enrichment protects against the effects of chronic stress on cognitive and morphological measured of hippocampal integrity. Neurobiol Learn Mem 2012;97:250-60.

48. Seong HH, Park JM, Kim YJ. Antidepressive effects of environmental enrichment in chronic stress-induced depression in rats. Biol Res Nurs 2018;20:40-8.

49. Shilpa BM, Bhagya V, Harish G, et al. Environmental enrichment ameliorates chronic immobilisation stress-induced spatial learning deficits and restores the expression of BDNF, VEGF, GFAP and glucocorticoid receptors. Prog Neuropsychopharmacol Biol Psychiatry 2017;76:88-100.

50. Thériault RK, Perreault ML. Hormonal regulation of circuit function: sex, systems and depression. Biol Sex Differ 2019;10:12.

51. McFadden LM, Paris JJ, Mitzelfelt MS, et al. Sex-dependent effects of chronic unpredictable stress in the water maze. Physiol Behav 2011;102:266-75

52. Bjork MH, Sand T, Brathen G, et al. Quantitative EEG findings in patients with acute, brief depression combined with other fluctuating psychiatric symptoms: a controlled study from an acute psychiatric department. BMC Psychiatry 2008;8:89.

53. Morgan ML, Witte EA, Cook IA, et al. Influence of age, gender, health status, and depression on quantitative EEG. Neuropsychobiology 2005;52:71-6.

54. McVoy M, Aebi ME, Loparo K, et al. Resting-state quantitative electroencephalography demonstrates differential connectivity in adolescents with major depression disorder. J Child Adolesc Psychopharmacol 2019;29:370-7.

55. Saletu B, Brandstatter N, Metka M, et al. Hormonal, syndromal and EEG mapping studies in menopausal syndrome patients with and without depression as compared with controls. Maturitas 1996;23:91-105.

56. Saletu B, Brandstatter N, Metka M, et al. Double-blind, placebocontrolled, hormonal, syndromal and EEG mapping studies with transdermal oestradiol therapy in menopausal depression. Psychopharmacology (Berl) 1995;122:321-9.

57. Shim M, Im CH, Kim YW, et al. Altered cortical functional network in major depression disorder: a resting-state electroencephalogram study. Neuroimage Clin 2018;19:1000-7.

58. Flor-Henry P, Lind JC, Koles ZJ. A source-imaging (low-resolution electromagnetic tomography) study of the EEGs from unmedicated males with depression. Psychiatry Res 2004;130:191-207.

59. Pizzagalli DA, Oakes TR, Davidson RJ. Coupling of theta activity and glucose metabolism in the human rostral anterior cingulate cortex: an EEG/PET study of normal and depressed subjects. Psychophysiology 2003;40:939-49.

60. Iseger TA, Padberg F, Kenemans JL, et al. Neuro-cardiac-guided TMS (NCG-TMS): probing DLPFC-sgACC-vague nerve connectivity using heart rate-first results. Brain Stimul 2017;10:1006-8.

61. Hakami T, Jones NC, Tolmacheva EA, et al. NMDA receptor hypofunction leads to generalized and persistent aberrant gamma oscillations independent of hyperlocomotion and the state of consciousness. PLoS One 2009;4:e6755.

62. Hunt MJ, Raynaud B, Garcia R. Ketamine dose-dependently induces high-frequency oscillations in the nucleus accumbens in freely moving rats. Biol Psychiatry 2006;60:1206-14

63. Gazit T, Friedman A, Lax E, et al. Programmed deep brain stimulation synchronizes VTA gamma band field potential and alleviates depressive-like behaviour in rats. Neuropharmacology 2015;91:135-41.

64. Crowell AL, Riva-Posse P, Holtzheimer PE, et al. Long-term outcomes of subcallosal cingulate deep brain stimulation for treatmentresistant depression. Am J Psychiatry 2019;176:949-56.

65. Marmor O, Valsky D, Joshua M, et al. Local vs. volume conductance activity of field potentials in the human subthalamic nucleus. J Neurophysiol 2017;117:2140-51.

66. Larsen MH, Mikkelsen JD, Hay-Schmidt A, et al. Regulation of brain-derived neurotrophic factor (BDNF) in the chronic unpredictable stress rat model and the effects of chronic antidepressant treatment. J Psychiatr Res 2010;44:808-16. 